\title{
Managing The Nondeflating Urethral Catheter
}

\author{
CPT Andrew J. Shapiro, MC USA; MAJ Douglas W. Soderdabl, MC USA; \\ LTC Richard S. Stack, MC USA; and LTC James H. North Jr, MC USA
}

Background: Urethral catheterization is a routinely performed procedure in hospitalized patients. Numerous complications have been reported secondary to urethral catheter placement.

Metbods: The medical literature was searched using the following key words: "urethral catheter," "complications," and "management." A case report is described and a review of the literature is provided to assist in managing the nondeflating urethral catheter balloon.

Results and Conclusions: All physicians who order urethral catheters must be aware of the possibility of a nondeflating catheter balloon and be comfortable with its initial management. The literature has an abundance of techniques for managing the nondeflating urethral catheter balloon. The approach and algorithm provided serve as a guide for the management of this complication by the family physician.(J Am Board Fam Pract 2000;13:116-9.)

One of the most commonly performed invasive procedures in hospitalized patients is urethral catheterization with a Foley catheter. ${ }^{1}$ It has been estimated that $10 \%$ to $15 \%$ of hospitalized patients will undergo Foley catheter drainage at some time during their admission. ${ }^{2}$ Foley catheters are used routinely for both short- and long-term drainage of the urinary bladder and have been associated with a great many complications including infections, catheter encrustation, catheter blockage, bladder spasms, balloon rupture, leakage, and retained catheter. ${ }^{3}$ These complications occur more commonly with chronic indwelling catheters but can happen with short-term use as well. The retained Foley catheter is a vexing problem that many physicians are likely to encounter by during their careers. The purpose of this article is to review the methods available for the removal of retained Foley catheters caused by balloons that will not deflate and provide the family physician with strategies for managing this problem.

\section{Methods}

A case report of a patient with a nondeflating Foley urethral catheter balloon is described. The medical

Submitted, revised, 2 March 1999.

From the General Surgery Service (AJS), Urology Service (DWS, RSS), and Surgical Oncology JHN Jr), Eisenhower Army Medical Center, Fort Gordon, Ga. Address reprint requests to CPT Andrew J. Shapiro, MD, General Surgery Resident, General Surgery Service, Eisenhower Army Medical Center, Fort Gordon, GA 30905. literature on complications of urethral catheterization was reviewed using the following key words: "urethral catheter," "complications," and "management." An algorithm is constructed to assist the family physician in managing this complication of the nondeflating urethral catheter balloon.

\section{Case Illustration}

A 72-year-old man with a history of coronary artery disease and hypertension was admitted to the Family Practice service complaining of a several hour history of shortness of breath. He reported that he had run out of his "water pill" several days ago, and since that time he had noted increased swelling around his ankles. He was admitted with a diagnosis of congestive heart failure, and a Foley catheter was placed to monitor adequacy of diuresis. After 2 days of diuretic therapy, his shortness of breath and pedal edema had resolved. The Foley catheter was no longer needed; however, it could not be removed because the balloon would not deflate.

\section{Discussion}

Each part of the Foley catheter can be implicated as a potential cause of the balloon not deflating. The inflation valve can malfunction, thereby preventing return of the fluid filling the balloon. External clamping, crushing, or kinking can damage the inflation channel, or it can become obstructed by solute crystallization when fluids other than sterile water are utilized for balloon inflation. Regardless 
Cut balloon port proximal to inflation valve

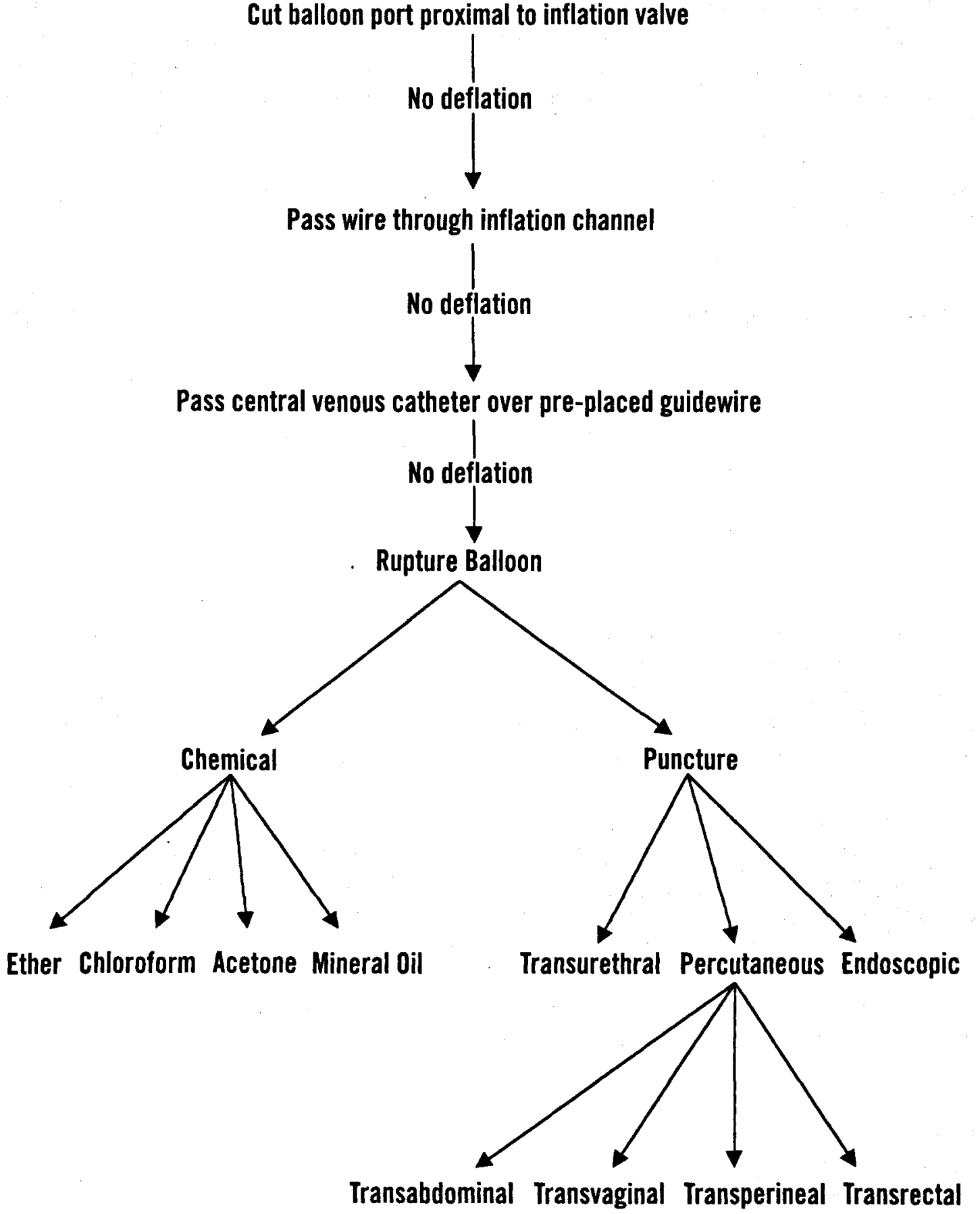

Figure 1. Management algorithm for the nondeflating urethral catheter balloon.

of the cause, the family physician can initially manage the challenge of the nondeflating Foley balloon. If initial measures fail, the guidance and assistance of a urologist should be sought.

Many sources are available describing methods and techniques used to remove Foley catheters when the balloon will not deflate. We propose a simple algorithm the family physician can use to assist in the management of the nondeflating Foley catheter balloon (Figure 1).

The initial step in the management of the nondeflating Foley catheter balloon is to advance the catheter to be sure the balloon is within the bladder. If this maneuver fails to deflate the balloon, the balloon port should be cut proximal to the inflation valve. ${ }^{4}$ This step eliminates the valve mechanism 
and should allow the fluid to drain freely from the balloon. If this method is unsuccessful, the obstruction is most likely along the length of the catheter or at the entrance to the balloon. ${ }^{5}$ Although this technique might not be successful at deflating the balloon, removal of the valve facilitates additional deflation techniques.

The next step is to pass a lubricated fine-gauge guidewire, such as a stylet, through the inflation channel after the valve mechanism has been severed. ${ }^{6}$ The guidewire might relieve any obstruction or allow the fluid to egress along the wire, thereby draining the balloon.

If the stylet technique is not adequate at draining the balloon, a well-lubricated 22-gauge central venous catheter can be inserted over the preplaced guidewire using the Seldinger technique. ${ }^{7}$ When the catheter tip is advanced enough to be in the balloon, the guidewire is removed, and the balloon should drain. If the balloon does not drain, the wire may be reinserted and used to advance the second catheter farther, as necessary.

If all these attempts at balloon deflation fail, techniques to rupture the balloon are available to facilitate catheter removal. Hyperinflation with air or saline should be avoided because of the painful nature of the procedure, risk of bladder rupture, and need for further treatment as a result of retained balloon fragments.

Several chemicals have been used to dissolve the balloon wall and thereby allow its deflation. Ether, chloroform, acetone, and mineral oil are among the agents most commonly used. ${ }^{8,9}$ Unfortunately, exposure of the bladder urothelium to these chemicals can result in chemical cystitis, bladder contractures, hematuria, bladder rupture, and death. ${ }^{10}$ In addition, balloon fragments might be retained within the bladder, predisposing the patient to a variety of complications, including calculus formation, ${ }^{11}$ recurrent urinary tract infections, ${ }^{12}$ and irritation when voiding. ${ }^{2}$ If chemicals are to be used to dissolve the balloon, the bladder should be filled through the irrigation-drainage port with $200 \mathrm{~mL}$ of sterile water or normal saline to dilute the chemical when it enters the bladder as the balloon ruptures.

Mineral oil is recommended for this procedure because it is less toxic than the other substances and has an $85 \%$ to $90 \%$ success rate with no adverse effects. ${ }^{8}$ About $10 \mathrm{~mL}$ of mineral oil can be injected through the inflation port. If the desired effect does not occur within 15 minutes, an additional $10 \mathrm{~mL}$ of mineral oil can be injected with the expectation that the balloon will rupture within 30 minutes. If this technique is successful, the physician must inspect the catheter carefully on removal. If any possibility of a retained balloon fragment exists, cystoscopy is warranted for evaluation and prompt removal.

Balloon rupture can also be accomplished by puncture with a sharp instrument. Several routes are available, including transurethral, percutaneous, and endoscopic. Although not appropriate for men, the transurethral approach should be attempted first in women. Steady, continuous traction on the catheter might cause part of the balloon to show through the urethral meatus, and the balloon can then be pierced with a lumbar needle. ${ }^{9}$ Another transurethral method involves the pericatheter passage of an angiographic catheter needle protected by the outer sheath. When the balloon is reached, the needle can be advanced to puncture the balloon. ${ }^{4}$

Foley catheter balloons can also be punctured percutaneously using transabdominal, transvaginal, transperineal, and transrectal approaches. Because these methods are more invasive, they should be performed as described only with the assistance of a urologist or radiologist using sonographic guidance. The transvaginal technique involves inserting a spinal needle through the anterior vaginal wall while the catheter is held on traction, positioning the balloon at the bladder neck. ${ }^{5}$ The transperineal approach can be used in men or women. The needle is passed through the perineum while the balloon is palpated through the rectum in men and either through the rectum or the vagina in women. ${ }^{13}$ Transperineal puncture has also been performed using transrectal sonographic guidance. ${ }^{14}$

Transabdominal balloon puncture has been performed blindly by passing a spinal needle or suprapubic catheter puncture needle through the suprapubic abdominal wall. ${ }^{15}$ The needle is advanced and aimed toward the bladder neck while the catheter is held on traction. Although we do not recommend the blind transabdominal approach because of the serious risk of injury to abdominal structures, this route is safe and effective when combined with sonographic guidance. ${ }^{16}$

We recommend the transrectal route for percutaneous balloon puncture in men. With the aid of digital rectal palpation, the balloon can be pierced 
blindly; ${ }^{13}$ however, transrectal sonography is more commonly used to assist with transrectal balloon puncture. ${ }^{17}$ In cases of transrectal needle puncture, the patient should be treated prophylactically with intravenous antibiotics and an enema.

Although the above methods are often effective, they are not uniformly reliable. If the techniques described above are not successful at deflating the Foley catheter balloon, a urologist should be consulted to perform endoscopic balloon puncture. This method is the most reliable available because it is performed under direct visualization. The physician has the added benefit of being able to evaluate bladder injury and inspect for retained fragments at the time of catheter removal. Numerous techniques using rigid and flexible cystoscopy have been described for endoscopic balloon puncture. The catheter can be cut at the meatus and pushed into the bladder using the cystoscope. The balloon can then be punctured and deflated by injection needles passed through the working channel of the cystoscope. ${ }^{18}$

\section{Conclusion}

Numerous techniques have been described that are effective in removing a Foley catheter balloon that will not deflate. We believe that the family physician can safely perform all the described techniques before resorting to endoscopic deflation, although some physicians might require radiologic support. Physicians not comfortable with invasive procedures might wish to consult a urologist for those techniques. We also strongly advise consulting a urologist with all cases of suspected balloon rupture so that cystoscopy can be performed to assure that no balloon fragments are retained. We hope this article provides the clinician with an armamentarium to manage the nondeflating Foley catheter balloon.

\section{References.}

1. Cancio LC, Sabanegh ES Jr, Thompson IM. Managing the Foley catheter. Am Fam Physician 1993; 48:829-36.
2. Williams JC, Doebler RW, Curtis MR, Richardson JR. Deflation techniques for faulty Foley catheter balloons: presentation of a cystoscopic technique. Tech Urol 1996;2:174-7.

3. Ndirangu K, Ngugi M. Problems of indwelling Foley catheters. East Afr Med J 1994;71:132-4.

4. Carr LK. An alternative to manage a nondeflating Foley catheter in women. J Urol 1995;153(3 Pt 1): 716-7.

5. Kleeman FJ. Technique for removal of Foley catheter when balloon does not deflate. Urology 1983; 21:416.

6. Vandendris $M$. How to deflate refractory balloon of a bladder catheter. Urology 1985;26:300.

7. Lutin CD, Mullins SD. Deflation of a Foley catheter balloon. N Engl J Med 1982;307:1270.

8. Murphy GF, Wood DP Jr. The use of mineral oil to manage the nondeflating Foley catheter. J Urol 1993;149:89-90.

9. Chin PL, Singh RK, Athey G. Removal of retained urinary catheters. Br J Urol 1984;56:185-7.

10. Thomas A, Bhat S, Mathew JK. An easy method to remove self-retained Foley catheter. J Indian Med Assoc 1994;92:348.

11. Ohashi $H$. [A case of a bladder calculus due to a ruptured balloon fragment of a Foley catheter.] Hinyokika Kiyo 1997;43:227-8.

12. Eckford SD, Persad RA, Brewster SF, Gingell JC. Intravesical foreign bodies. Five-year review. $\mathrm{Br} \mathrm{J}$ Urol 1992;69:41-5.

13. Anyanwu SN, Apakama IG. Simple methods for the removal of non-deflating balloon bladder catheters suitable for the developing countries. Trop Doct 1991;21:84-5.

14. Walters NA, Kilbey J, Rickards D. A technique for the removal of retained balloon bladder catheters. Br J Radiol 1988;61:320-1.

15. Adebamowo CA, Okeke LI. The retained urinary catheter. Trop Geogr Med 1993;45:186-8.

16. Naunton-Morgan TC, Barrett NK, Boultbee JE. Simple procedure for the removal of a non-deflating balloon bladder catheter: two cases. Br J Radiol 1986; 59:1043-4.

17. Collins GN. Catheter balloon ruprure using transrectal ultrasound. J Urol 1993;149:91.

18. Goel MC, Bhadauria RP, Banerjee GK, Kapoor R. A new endoscopic technique for non-deflating Foley balloon. Arch Esp Urol 1995;48:973-5. 